\title{
Episodic Hypoglycemia with $\psi$-Hydroxy Fatty Acid Excretion
}

\author{
ELEANOR COLLE, ${ }^{(31)}$ O.A. MAMER, J.A. MONTGOMERY, AND JOHN D. MILLER \\ Montreal Children's Hospital-McGill University Research Institute and the McGill University-Medical Research \\ Council of Canada Biomedical Mass Spectrometry Unit, Montreal, Quebec, Canada
}

\begin{abstract}
Summary
We present case histories of two young children with episodes of hypoglycemia, elevation of SGOT, low insulin levels, increased urinary excretion of $\psi$-hydroxy fatty acids (5-hydroxyhexanoic, 7 hydroxyoctanoic and 9-hydroxydecanoic), traces of the corresponding $\psi$-ketoacids and elevations of urinary adipic, suberic, and sebacic acids. The ratio of $\psi$-hydroxy fatty acids to 3-hydroxybutyric in the urine of these patients is higher than in patients of similar ages with similar illnesses. These acids persisted while the patients were well. Increased urinary $\psi$-hydroxy fatty acids could be reproduced by a load of medium chain triglycerides without precipitating other clinical symptoms. Three children with hypoglycemia were found not to excrete measurable amounts of these unusual acids while ill. A medium chain triglyceride load in one of these children after recovery failed to elicit $\psi$-hydroxy acid excretion. Small amounts of urinary 5-hydroxyhexanoic acid only were found in two patients with acute Reye's syndrome and in three of five severely ill children with starvation ketonuria. In this last group, no urinary $\psi$-hydroxyacids could be detected after recovery. Normal children do not excrete measurable amounts (less than 1 $\mathrm{mg} / \mathrm{g}$ creatinine) of these $\psi$-hydroxyacids.
\end{abstract}

\section{Abbreviations}

GC-MS, gas chromatography-mass spectrometry

JVS, Jamaican vomiting sickness

LCT, long chain triglyceride

LDH, lactic dehydrogenase

MCT, medium chain triglyceride

SGOT, serum glutamic oxalic transaminase

SGPT, serum glutamic pyruvic transaminase

$\psi$-hydroxy acid, two homologous series of fatty acids have been detected and studied for the first time in two patients with an apparent defect of fatty acid catabolism. The first series are fatty acids that are hydroxylated on the next-to-last carbon atom (as numbered from the carboxyl end). The second series are the corresponding keto-acids formed (presumably) by further oxidation of the hydroxyl substituted carbon atom. It is proposed that these acids be termed $\psi$-hydroxy and $\psi$-keto acids ( $\psi$ : Psi, the next-to-last letter of the Greek alphabet) in keeping with the accepted convention that $\alpha, \beta$, and $\omega$ designate functionality on the first carbon, second carbon, and the last carbon atom remote from the carboxyl carbon respectively.

Many well-defined metabolic, hormonal, and toxic disorders can result in symptomatic hypoglycemia in children. Plasma insulin levels are used to divide these episodes into two groups. Hypoglycemic episodes with low insulin levels are usually due to blocks in the metabolic pathways of glycolysis and/or gluconeogenesis. They occur during periods of caloric deprivation, are characterized by increased fatty acid metabolism, and are clinically manifest by increased ketone body formation and excretion.
Those episodes with high insulin levels are due to increased glucose utilization and decreased hepatic glucose output secondary to inappropriate insulin secretion which, in addition, inhibits lipolysis. Hence hypoglycemia with hyperinsulinemia usually occurs in the absence of ketonuria.

Some children, however, have single or recurrent episodes of hypoglycemia, that remain unexplained even after elimination of all of the well recognized causes. Recently, it has been recognized that defects in fatty acid utilization may present in childhood as hypoglycemia. Depending on the location of the metabolic block, ketone body formation may be either absent or decreased. The investigation of the organic acid patterns in serum and urine from such individuals promises new insights into these 'idiopathic' hypoglycemic states. In a preliminary report $(20)$ we described an unusual urinary organic acid profile found for an infant with episodic hypoglycemia. The most unusual features were two homologous series of $\psi$-hydroxy and $\psi$-keto fatty acids, which were interpreted to be consistent with an error in the $\beta$-oxidation of medium chain fatty acids. We wish to report the results of further study of this patient and the study of a second unrelated patient found with apparently the same disorder. We contrast the organic acid profiles in these children with those found in normoglycemic children without ketosis, children with hypoglycemia, children with ketonuria, and in four patients with Reye's syndrome.

\section{MATERIALS AND METHODS}

Case histories. Patient 1, the second child of unrelated healthy parents, was 15 months of age at the time of her first admission. She was born after a normal pregnancy, weighed $2.81 \mathrm{~kg}$ and measured $50.2 \mathrm{~cm}$. Mental and motor development had been normal. The parents had moved into a new apartment, the floors of which had been refinished with urethane $1 \mathrm{wk}$ before admission. The child had not been off the premises unaccompanied. There was no known spraying with insecticides in the area. On the evening before admission, the infant ate dinner, but vomited later in the evening and throughout the night. She arrived in the emergency room at $1425 \mathrm{~h}$. Her height was $75 \mathrm{~cm}$ (25th centile) and weight $8 \mathrm{~kg}$ ( $3 \mathrm{rd}$ centile). Temperature was $38.5^{\circ} \mathrm{C}$, pulse 80 , blood pressure 75 systolic by palpation. She was unresponsive except to pain and had a high pitched cry. The liver was felt $2 \mathrm{~cm}$ below the right costal margin. The blood glucose was $6 \mathrm{mg} / \mathrm{dl}$, the urea nitrogen $40 \mathrm{mg} / \mathrm{dl}$ and the creatinine $0.8 \mathrm{mg} / \mathrm{dl}$. Sodium, potassium, chloride, and calcium were normal. The urine contained moderate amounts of ketones. Plasma cortisol was 25 $\mu \mathrm{g} / \mathrm{dl}$ and insulin less than 6 milliunits/liter. Blood ammonia was $26 \mu \mathrm{g} / \mathrm{dl}$. The glucose concentration of spinal fluid was $11 \mathrm{mg} / \mathrm{dl}$ and the protein concentration $13 \mathrm{mg} / \mathrm{dl}$. The child regained consciousness after receiving $12.5 \mathrm{~g}$ of glucose intravenously, but remained irritable for the next 2 days.

SGOT the day after admission was 86 units/liter. The amino acid chromatogram of urine was normal. Blood and spinal fluid cultures were negative. Culture of urine grew 100,000 colonies of $E$. coli. She received sulfisoxazol for her urinary tract infection. 
Two wk later the glucose concentration in plasma was $67 \mathrm{mg} / \mathrm{dl}$ and the free fatty acids $510 \mu \mathrm{M}$ after a fast of $16 \mathrm{~h}$ duration. At that time the administration of glucagon, $0.01 \mathrm{mg} / \mathrm{kg}$ intramuscularly, produced a maximum glucose value of $139 \mathrm{mg} / \mathrm{dl}$. The SGOT was 35 units/liter and the LDH 457 units/liter. Growth hormone values during the glucagon test were greater than $5 \mathrm{ng} /$ $\mathrm{ml}$. Examination of acute and convalescent sera showed no rise in antibody titer against adenovirus or reovirus.

The child was well until 8 months later when she again vomited and refused further feeds. She slept through the night, but did not arouse normally in the morning. She arrived at hospital at $0630 \mathrm{~h}$ and suffered a respiratory arrest shortly after arrival. Blood glucose was $6 \mathrm{mg} / \mathrm{dl}$, hydrogen ion concentration $36 \mathrm{nM}$, bicarbonate ion concentration was $24.5 \mathrm{mM}, \mathrm{PCO}_{2}$ was $35 \mathrm{mmHg}$. Physical examination revealed an unresponsive child; the liver edge was $1 \mathrm{~cm}$ below the costal margin. She awoke after infusion of glucose. LDH values were slightly elevated; SGOT levels were normal on days 1,3 , and 5 , but rose on day 7 to 103 units/liter. She has been well since the second episode. Her psychomotor development is normal.

Patient 2, a 16-month-old female, was the younger of two children. She was born at term weighing $3.18 \mathrm{~kg}$. There were no prenatal nor perinatal problems. Her nonrelated parents and older sister were in good health. She was admitted to hospital after a 24 $\mathrm{h}$ history of vomiting and fever $\left(38.5^{\circ} \mathrm{C}\right)$. Examination revealed a height of $78 \mathrm{~cm}$ (50th percentile), a weight of $8.8 \mathrm{~kg}$ (10th percentile). The child was somnolent and reacted only to pain. There were several small mouth ulcers present. The following day the liver was palpable $1 \mathrm{~cm}$ below the right costal margin. On admission the blood glucose was $34 \mathrm{mg} / \mathrm{dl}$, the urea nitrogen 21 $\mathrm{mg} / \mathrm{dl}$ and the electrolytes were normal. The spinal fluid glucose was $25 \mathrm{mg} / \mathrm{dl}$, the protein concentration $13 \mathrm{mg} / \mathrm{dl}$. The urine contained a small amount of ketones. The child became alert after intravenous glucose. The following day SGOT was 163 units/liter, SGPT 556 units/liter, LDH 455 units/liter (normal less than 360) and alkaline phosphatase 178 units/liter. Blood and spinal fluid cultures were negative. Five days post admission, the glucose was normal $(71 \mathrm{mg} / \mathrm{dl})$ and rose appropriately after glucagon stimulation $(110 \mathrm{mg} / \mathrm{dl})$. Her mental and physical development have continued to be appropriate for her age. At present she has no hepatomegaly and liver function tests are normal.

Methods. Glucose was determined with the Beckman Glucose Analyzer, total free fatty acids by a colorimetric method (18), ammonia by an ion-exchange resin method (Hyland Kit, Costa Mesa, CA), and hormonal levels by radioimmunoassay (14). The organic acids in urine and deproteinized serum were analyzed by coupled gas chromatography-mass spectrometry (GC-MS) (19). The sulphosalicylic acid precipitation and ether extraction method used here discriminates heavily against free fatty acids bound to protein and allows for the determination of the relatively much smaller fraction (approximately 5\%) remaining in free solution. Medium chain triglyceride loads were given to both patients as MCT oil (71\% octanoic acid, $1 \mathrm{~g} / \mathrm{kg}$ body weight) after an 8 -h fast. A long chain triglyceride load was given to only patient 1 as corn oil ( $40 \%$ linoleic, $45 \%$ oleic, $1 \mathrm{~g} / \mathrm{kg}$ body weight), after a similar fast.

In the first patient we examined the organic acids in urine excreted during and just after the spontaneous hypoglycemic episodes, and $6 \mathrm{~h}$ after the triglyceride loadings when in good health. Organic acids were also determined in deproteinized sera taken $3 \mathrm{~h}$ after second admission and $3 \mathrm{~h}$ after the MCT and LCT loadings. In the second patient, we examined urines collected 5 days after the hypoglycemic episode and $6 \mathrm{~h}$ after an MCT loading in good health. The urinary organic acid profiles of these two patients were compared to those of four contrasting groups: Group A (patients 3-9) were normoglycemic and had no acute illness; Group B (patients 10-12) had documented hypoglycemia; Group C (patients 13-17) were acutely ill and had ketonuria with normoglycemia, and Group D (patients 18-21) were in the acute phase of Reye's syndrome. Triglyceride loads $(1 \mathrm{~g} / \mathrm{kg})$ were made to several patients in Groups B and C following recovery: two in
$B$ and one in $C$ received LCT loads, whereas one in $B$ received an MCT load. Urinary organic acid profiles were then re-examined for these four patients and for two others in $\mathrm{C}$ without loads following their recovery.

The identities of the $\psi$-hydroxy and $\psi$-keto acids were confirmed by comparison of their mass spectra and retention times with those of the authentic acids obtained through synthesis (20). The mass spectrum of the bis-trimethylsilyl derivative of 5-hydroxyhexanoic acid was identical to that reported by Lawson and Chalmers (17).

\section{RESULTS}

Table 1 summarizes all the urinary acid data for the patients and the contrasting and control groups. Examination of the urine from patient 1 at the time of the first admission showed increased amounts of suberic and adipic acids (145 and $80 \mathrm{mg} / \mathrm{g}$ creatinine respectively). Two most unusual acids were also found, which proved to be 5-hydroxyhexanoic acid and 7-hydroxyoctanoic acid (concentration: $70 \mathrm{mg}$ and $30 \mathrm{mg} / \mathrm{g}$ creatinine, respectively).

Analysis of the first urine from the second admission (Fig. 1) revealed elevated quantities of 5-hydroxyhexanoic, 7-hydroxyoctanoic, 9-hydroxydecanoic, 5-ketohexanoic, and 7-ketooctanoic acids, along with adipic, suberic, and sebacic acids. Admission urine from the second patient was not available for analysis. The first available urine, taken 5 days after admission, had increased concentrations of 5-hydroxyhexanoic, 7-hydroxyoctanoic, octanoic, adipic, suberic and sebacic acids.

In contrast to patients 1 and $2, \psi$-hydroxyacid excretion did not occur in acute hypoglycemic Group B patients and was found in relatively small amounts (only as 5-hydroxyhexanoic acid) in three of five acute ketonuric Group $\mathrm{C}$ patients. Two of four patients with Reye's syndrome and microvesicular fatty infiltration of the liver on biopsy demonstrated urinary 5-hydroxyhexanoic acid but no other $\psi$-hydroxy or $\psi$-keto acids were detectable in their urines at any time. One of them died from severe encephalopathy and liver failure.

Loadings with MCT in both patients when healthy produced increases in urinary octanoic, 7-hydroxyoctanoic, suberic, and sebacic acids after $6 \mathrm{~h}$ that are generally very much more pronounced than a similar load in a Group B patient (No. 11) when healthy. The LCT loadings in patient 1 and in the two Group B patients (Nos. 10 and 12) were very much less effective in stimulating excretion of these acids than were the MCT loadings. When LCT was given to patient 15 after recovery from his severe gastroenteritis, there was only minimal excretion of adipic and pimelic acids.

The seven normal children in Group A did not excrete any of the $\psi$-hydroxy or $\psi$-ketoacids in measurable amounts (less than 1 $\mathrm{mg} / \mathrm{g}$ creatinine). When reexamined after recovery from their acute illness, Group C patients 16 and 17 had normal urinary acid profiles. After apparent recovery, patients 1 and 2 continued to excrete abnormally high concentrations of these $\psi$-hydroxy and dicarboxylic acids.

The ratios of the urinary concentrations of $\psi$-hydroxy acids to 3 -hydroxybutyric acid was chosen as a possible relative measure of the degree of inhibition of $\beta$-oxidation of medium chain fatty acids. Restriction of this pathway will reduce the rate of conversion of the medium chain acids to ketone bodies and thus raise these ratios. Unrestricted through-put on the other hand should reduce the tendency to accumulate $\psi$-hydroxy acids and facilitate 3hydroxybutyrate synthesis (i.e., reduce the ratios). This trend is clearly seen in Table 1 in Groups B and C where 3-hydroxybutyrate excretion is greatly elevated with respect to the $\psi$-hydroxyacids and is reflected in low ratios. Patients 1 and 2 have elevated $\psi$-hydroxyacids and hence have ratios greater than 1 , even when well. Group D patients without elevations of either 3-hydroxybutyrate or $\psi$-hydroxyacids have intermediate ratios.

GC-MS analysis of serum (deproteinized with sulphosalicylic acid) taken $3 \mathrm{~h}$ after the second admission of patient 1 showed accumulations of the usual ketone bodies along with other acids not frequently seen in serum: octanoic, 7-hydroxyoctanoic, 5- 


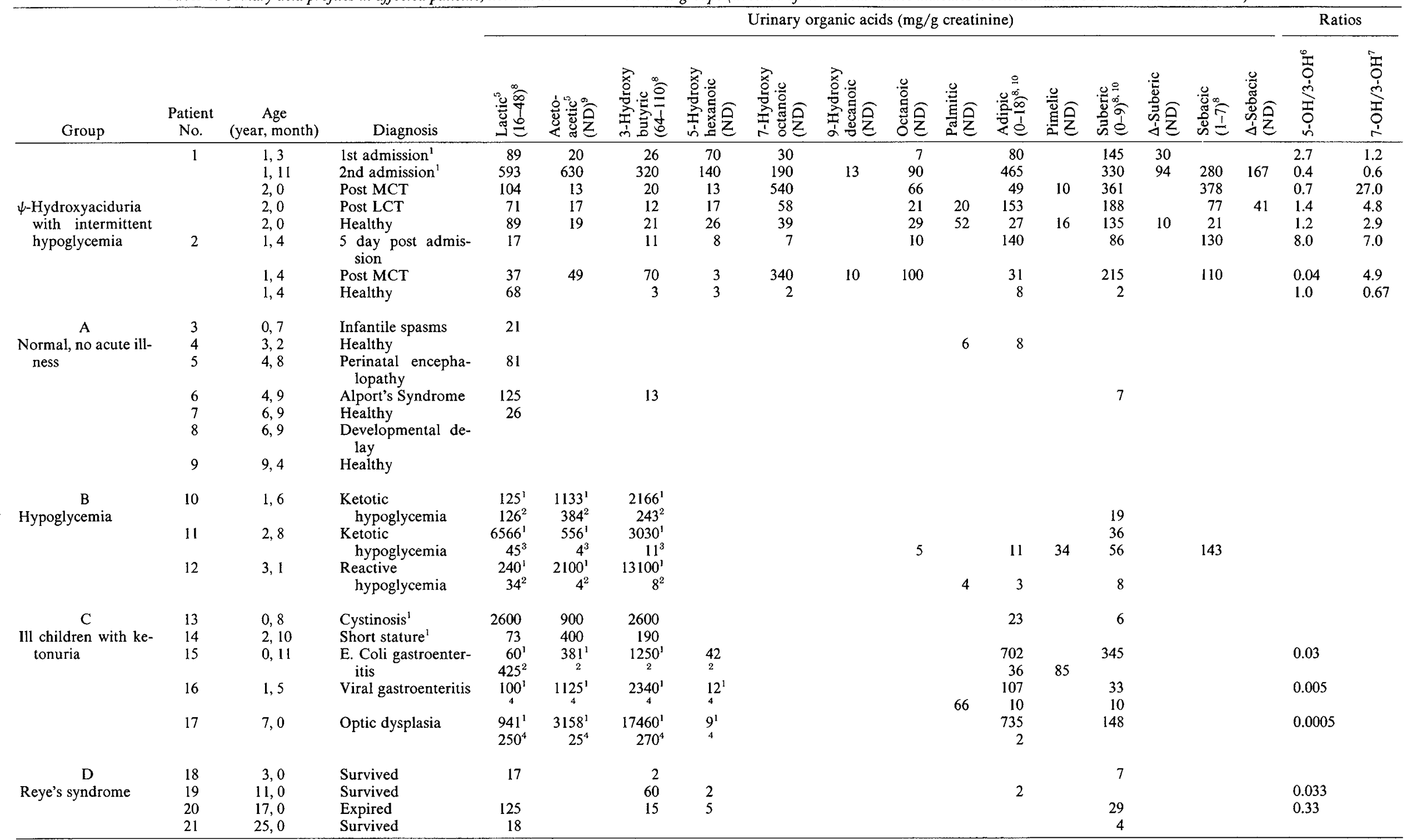

'Acutely ill.

${ }^{2}$ Long chain triglyceride challenge.

${ }^{3}$ Medium chain triglyceride challenge.

${ }^{4}$ Repeat profile on normal diet.

${ }^{5}$ Lactic and acetoacetic acid concentrations are consistently low by this method; results indicate relative trends.

${ }^{6}$ Ratio of concentrations of 5-hydroxyhexanoic to 3-hydroxybutyric acid.

${ }^{7}$ Ratio of concentrations of 7-hydroxyoctanoic to 3-hydroxybutyric acid.

${ }^{8}$ Normal range (mg/g creatinine), Gregersen et al. (ref. 12).

${ }^{9} \mathrm{ND}$, not detectable.

${ }^{10}$ Normal range (mg/g creatinine), Dosman et al. (ref. 9). 


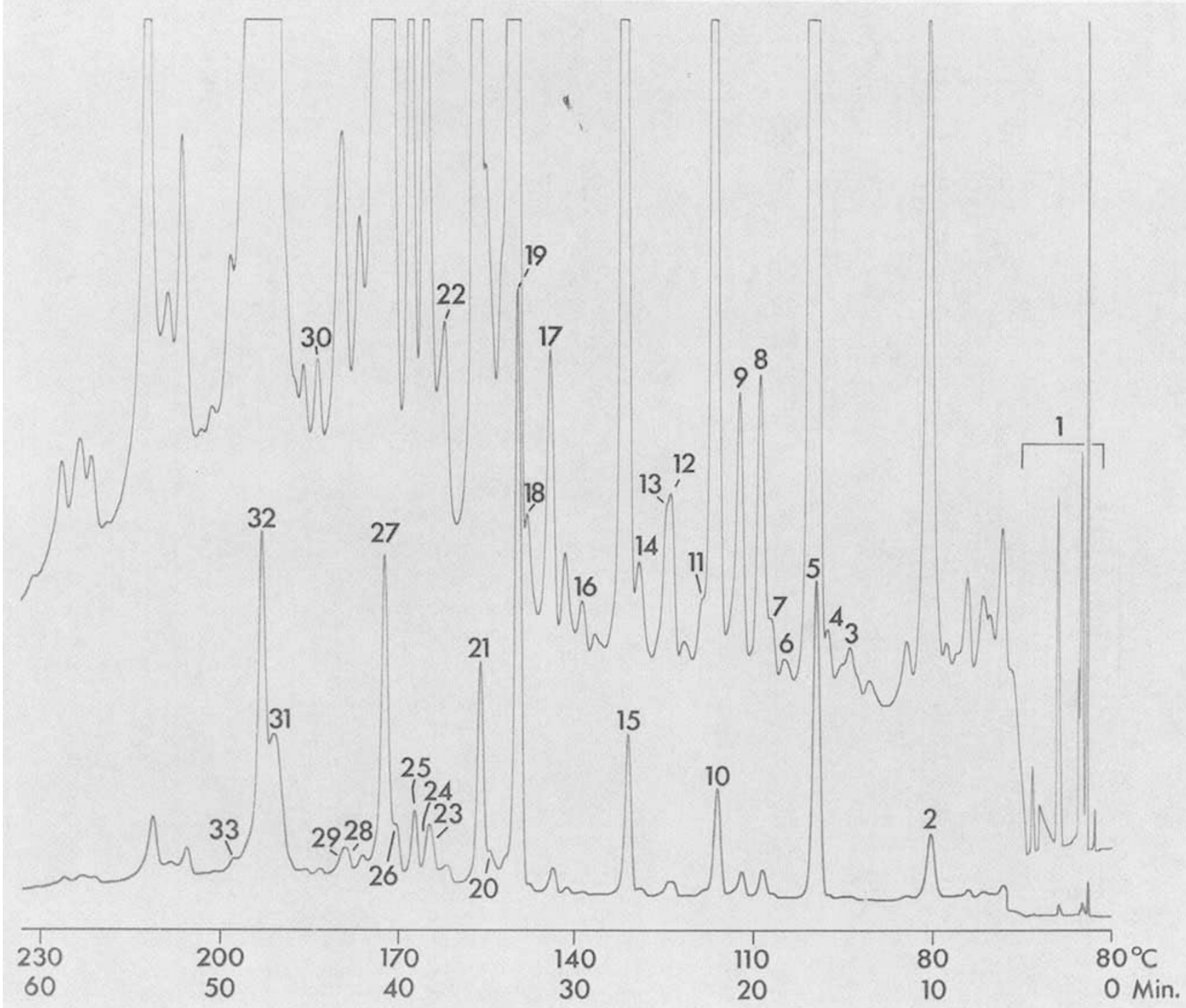

Fig. 1. Urinary organic acid profile for Patient 1 on second admission. The urine was treated with hydroxylamine hydrochloride to convert ketoacids to the more easily detected oxime acids (ref. 20). The peaks are identified as the trimethylsilyl derivatives of the following acids: 1 , derivatizing solvents and reagents; 2, lactic; 3,p-cresol; 4, pyruvic oxime; 5, 3-hydroxybutyric; 6, glyoxime; 7, 2-methyl-3-hydroxybutyric; 8, 3-hydroxy-isovaleric; 9, coeluting acetoacetic oxime and 2-ethylhydracrylic (3:1); 10, coeluting octanoic and acetoacetic oxime; 11, phenylacetic; 12, succinic; 13, 3-hydroxyhexanoic; 14, fumaric; 15, 5-hydroxyhexanoic; 16, possibly 6-hydroxyheptanoic (authentic sample unavailable); 17, 5-ketohexanoic oxime; 18, 3-hydroxyoctanoic; 19, adipic; 20, 5-hydroxymethyl-2-furoic; 21, 7-hydroxyoctanoic; 22, pimelic; 23, 7-ketooctanoic oxime; 24, p-hydroxy-phenylacetic; 25, 7-ketooctanoic oxime; 26, 2-octenedioic; 27, suberic; 28, 3-(p-hydroxyphenyl)propionic; 29, coeluting vanillic and homovanillic; 30, 9-hydroxydecanoic; 31, 2decenedioic; 32, sebacic; 33, 3-indoleacetic. The column used was $2 \mathrm{~m} \mathrm{x} 4 \mathrm{~mm}$ internal diameter glass packed with 6\% OV-101 on Chromosorb W HP and temperature programmed at $3 \mathrm{deg} / \mathrm{min}$ after an initial delay of $10 \mathrm{~min}$ at $80^{\circ} \mathrm{C}$. Each ketoacid forms a pair of oxime derivatives (E and $\mathrm{Z}$ ) that are gas chromatographically separable as the trimethylsilyl derivatives (e.g., peaks 9 and 10 , and 23 and 25 ). The ratio of the gains of the dual pen amplifiers has been set at 10:1.

hydroxyhexanoic, and adipic acids (Table 2). More remarkable, was the complete absence (less than $0.1 \mu \mathrm{M}$ ) of free (i.e., nonprotein bound) palmitic, oleic, and stearic acids, which are normally detected and easily quantitated in serum by this method. The MCT loading in patient 1 also produced serum elevations of octanoic, 7-hydroxyoctanoic, suberic and sebacic acids after $3 \mathrm{~h}$ that are very much larger than the values obtained both on admission and after the LCT loading (Table 2). Hypoglycemia was not detected during any of these loadings. Serum samples were not available from children in the contrast groups who underwent triglyceride loadings.

\section{DISCUSSION}

Several unusual features occurring together in our two patients distinguish them from others with related disorders: (1) episodic hypoglycemia; (2) large urinary concentrations of the $\psi$-hydroxyacids and traces of the $\psi$-ketoacids; (3) increased excretion of adipic, suberic, and sebacic acids, (4) decreased serum fatty acids (patient 1); and (5) increased ratios of 5-hydroxyhexanoic and 7hydroxyoctanoic acids to 3-hydroxybutyric acid.

In over 700 organic acid profiles done by GC-MS in our laboratory, 7-hydroxyoctanoic, 9-hydroxydecanoic, and the $\psi$-ketoacids have not been detected until the present study. 5-Hydroxyhexanoic acid can occasionally be detected in trace quantities in apparently normal urine (approximately $1 \mathrm{mg} / \mathrm{g}$ creatinine or less) and in small concentrations (typically $5 \mathrm{mg} / \mathrm{g}$ creatinine or less) in circumstances associated with disturbed lipid metabolism.

High concentrations of urinary dicarboxylic acids produced by increased $\omega$-oxidation are frequently noted in situations for example in which intense fatty acid oxidation is occurring (9) or after medium chain triglyceride loads (21). On the other hand, 
Table 2. Serum organic acids determined in patient 1 on second admission and $3 \mathrm{~h}$ after medium- (MCT) and long-chain triglyceride $(L C T)$ loadings ${ }^{1}$

\begin{tabular}{|c|c|c|c|c|}
\hline \multirow[b]{2}{*}{ Acid } & \multicolumn{4}{|c|}{ Concentration $(\mu \mathrm{M})$} \\
\hline & $\begin{array}{c}\text { 2nd } \\
\text { Admission }\end{array}$ & $\begin{array}{l}\text { Post } \\
\text { MCT }\end{array}$ & $\begin{array}{l}\text { Post } \\
\text { LCT }\end{array}$ & $\begin{array}{l}\text { Healthy } \\
\text { Controls }\end{array}$ \\
\hline 3-Hydroxybutyric & 135 & 56 & 33 & $50 \pm 39^{3}$ \\
\hline Acetoacetic & 78 & 3.5 & 5.9 & $20 \pm 20^{3}$ \\
\hline Octanoic & 14 & 46 & 6.3 & $\mathrm{ND}^{4}$ \\
\hline 5-Hydroxyhexanoic & 1.5 & $\mathrm{ND}^{5}$ & ND & ND \\
\hline 7-Hydroxyoctanoic & 1.3 & 19 & ND & ND \\
\hline Adipic & 2.7 & 2.0 & 6.9 & $0.14-0.34^{6}$ \\
\hline Suberic & 0.9 & 6.0 & 1.2 & $0.11-0.29^{6}$ \\
\hline Sebacic & 0.6 & 5.0 & $\mathrm{ND}$ & $0.05-0.15^{6}$ \\
\hline Lauric & 0.6 & 1.7 & $\mathrm{ND}$ & $\mathrm{NA}^{5}$ \\
\hline Palmitic & ND & 7.8 & 12 & $4.5 \pm 2.7^{7}$ \\
\hline Oleic, Linoleic & ND & 5.0 & 7.8 & $5.0 \pm 2.8^{7}$ \\
\hline Stearic & $\mathrm{ND}$ & 3.5 & 5.6 & $4.2 \pm 2.8^{7}$ \\
\hline
\end{tabular}

' Loadings did not reproduce the hypoglycemia noted on admission.

${ }^{2}$ Serum collected randomly from fed healthy children.

${ }^{3}$ Unpublished data; values used routinely as normal ranges in our laboratory.

${ }^{4}$ ND Not detectable (less than $0.1 \mu \mathrm{M}$ ).

${ }^{5}$ NA Not available.

${ }^{6}$ Values taken from N. Gregersen (ref. 12).

${ }^{7}$ Normal ranges used in our laboratory for these fatty acids remaining in solution after protein precipitation with sulphosalicylic acid.

conditions associated with decreased $\beta$-oxidation of fatty acids will also promote increased dicarboxylic acids in urine and blood. We have previously reported the presence of increased excretion of dicarboxylic acids by a child with carnitine deficiency (16), a condition in which transfer of acyl-CoA esters into mitochondria is impaired. During early childhood, hypoglycemia occurred in this patient. But long chain fatty acids accumulate in the serum of individuals with carnitine deficiency in contrast to the paucity of long chain fatty acids seen in the admission serum of patient 1 . Furthermore, we could not detect $\psi$-hydroxyacids in the patient with carnitine deficiency. These distinguishing features clearly suggest that our patients do not have carnitine deficiency.

The pattern of clinical symptoms in the present cases bears certain similarities to those reported by Tanaka et al (26) in two cases of Jamaican vomiting sickness (JVS). The hypoglycemia in JVS is due to a strong inhibition of several CoA dehydrogenases by methylenecyclopropylacetic acid, a metabolite of hypoglycine A found in the unripe akee bean. The urinary organic acid profiles in JVS and in our acute Patient 1 however are distinctly different. Excretion of 3-hydroxybutyric acid is higher in patient 1 during the acute attack than in either of the two JVS cases and higher than that reported in the child with carnitine deficiency. More importantly, in JVS, as in Glutaric Aciduria Type II, large amounts of ethylmalonic, glutaric, and isovaleric acids accumulate due to a generalized inhibition of dehydrogenases for butyryl CoA, glutaryl CoA, and isovaleryl CoA, respectively. An excellent review of the similarities in clinical and laboratory findings in these two disorders and others that are related has recently been published (27).

Lawson and Chalmers $(4,5,6,17)$ have reported finding 5hydroxyhexanoic acid, as well as increased quantities of adipic and suberic acids, in the urine specimens from twin male infants with profound hypoglycemia. They pointed out the similarities of the organic acid pattern to that seen in Jamaican vomiting sickness and hypothesized that the 5-hydroxyhexanoic acid might be the metabolic product of a hypoglycine analogue containing the 4alkenoic acid moiety necessary for the production of hypoglycemia. They speculated that berries from shrubs found in temperate zones contain such hypoglycine analogues. Our two patients are clinically similar to those reported by Lawson and Chalmers (6), by Gregersen et al. (13) and by Naylor et al. (23) but differ apparently most significantly from them by the presence of the two homologous series of $\psi$-hydroxy and $\psi$-ketoacids not reported by these authors. The occurrence of these two series in the acute phase and after an MCT load strongly argues that in our patients they are derived from fatty acid metabolism and not from an exogenous hypoglycine analogue, which is proposed by Lawson and Chalmers as the precursor of 5-hydroxyhexanoic acid and the precipitating cause of dicarboxylic aciduria in their patients. Tanaka has reported that in hypoglycine intoxication other acids not reported by Lawson and Chalmers (5) occur as a result of the generalized inhibition of CoA dehydrogenases. These observations lead us to believe that their patients may have had a disorder similar to that reported here and not related to an exogenous toxin.

The administration of sodium valproate, a $\mathrm{C}_{8}$ branched chain fatty acid, has also been associated with dicarboxylic aciduria (22), presumably on the basis of interference with fatty acid oxidation. A Reye's-like syndrome has also been associated with valproic acid therapy (10). Patients 1 and 2 have never been administered valproic acid to our knowledge.

We have seen one child in whom a diagnosis of recurrent Reye's syndrome had been made (28), and in whom the clinical picture of episodic hypoglycemia with mild abnormalities of liver function closely resembled that of the present cases. Unfortunately no studies of organic acid excretion during the hypoglycemic episodes were performed. The clinical similarities between Reye's syndrome and Jamaican vomiting sickness have been the subject of comment (8). Patients 1 and 2 do not satisfy the clinical criteria for the diagnosis of Reye's syndrome (7) as there was no history of a biphasic illness nor encephalopathy. Both became alert after intravenous glucose administration and the urinary and serum organic acid profiles of our two patients are markedly different from those of our cases of Reye's syndrome.

Gregersen et al. $(11,13)$ have reported two children with recurrent episodes of profound (and in one fatal) hypoglycemia, in whom urinary excretion of $\mathrm{C}_{6}, \mathrm{C}_{8}$, and $\mathrm{C}_{10}$ dicarboxylic acids occurred in conjunction with the excretion of the glycine conjugate of suberic acid. In one child, 5-hydroxyhexanoic acid was also found. They suggested a limit in $\beta$-oxidation of fatty acids as a cause of the attacks.

Urinary 5-hydroxyhexanoic, 7-hydroxyoctanoic, and the $\mathrm{C}_{6}-$ $\mathrm{C}_{10}$ dicarboxylic acids have very recently been reported (25) in premature and small-for-date infants fed approximately $8 \mathrm{~g} / \mathrm{kg}$ per day of MCT containing principally octanoic (49\%), decanoic (26.1\%), and linoleic (13.4\%) acids. 9-Hydroxydecanoic acid could not be detected; however, and octanoic and the $\psi$-keto acids were not reported. These babies were ingesting MCT in their formula at least $24 \mathrm{~h}$ before the study and hence received a considerably larger dose than did our patients 1 and 2 during their loading studies. They reported the total $\mathrm{C}_{6}-\mathrm{C}_{10}$ dicarboxylic acid level in urine as being $1333 \mathrm{mg} / \mathrm{g}$ creatinine. After the $1 \mathrm{~g} / \mathrm{kg} \mathrm{MCT}$ loading, patients 1 and 2 excreted lower levels of these acids, 788 and $356 \mathrm{mg} / \mathrm{g}$ creatinine, respectively. $\psi$-Hydroxy acid excretion in the premature infant study (25) equaled $251 \mathrm{mg} / \mathrm{g}$ creatinine as compared to 553 and $353 \mathrm{mg} / \mathrm{g}$ creatinine for patients 1 and 2 . Even though our patients received a smaller dose of MCT, they excreted considerably higher quantities of the $\psi$-hydroxyacids.

Glucose, free fatty acids, and ketone bodies (3-hydroxybutyric and acetoacetic acids) are the principal fuels of the body. Fatty acids are metabolized by a series of steps that include hydrolysis of the triglycerides, formation of the CoA esters, transport across the mitochondrial membrane via the carnitine acyl transferase reactions, and oxidation by a process by which 2 carbon fragments are removed from the carboxyl end of the fatty acid as acetyl CoA. Acyl dehydrogenase enzymes, which are probably specific for the various length carbon chains, are essential for this process. Acetyl CoA thus generated is a major substrate for formation of ketone bodies (acetoacetic and 3-hydroxybutyric acids).

A microsomal fraction of liver has been shown to catalyze the hydroxylation of the $\omega$ and $\psi$ carbon atoms of fatty acids and the further oxidation of the $\omega$-hydroxy acids to dicarboxylic acids ( 1 , 2 ). The ratios of $\omega$ to $\psi$-oxidized products of stearate, palmitate 
and laurate in vitro were 3,2 , and 1 respectively (1) and suggest that at still shorter chain lengths, $\psi$-hydroxylation may in fact compete well with $\omega$-oxidation. Under ordinary circumstances, $\omega$-oxidation plays a minor role (15) although it has been suggested that a possible function of $\omega$-oxidation is to detoxify fatty acids that might poison the respiratory chain $(2,13)$. Oxidation of the $\psi$-hydroxyacids to the corresponding $\psi$-ketoacids may occur by an enzyme system reported to convert 17-hydroxystearic acid to 17 ketostearic acid (3). We are not aware of any further metabolic use for these $\psi$-ketoacids.

The etiology of the disorder(s) affecting patients 1 and 2 is unknown; however, the fact that there is a series of $\psi$-hydroxyacids that are elevated when the patients are acute and that these observations are reproduced after an MCT load, points to a defect of fatty acid metabolism. We speculate that our two patients have a defect in the mitochondrial acyl-dehydrogenase enzyme specific for the 6-10 carbon fatty acid $\mathrm{CoA}$ esters, and are, therefore, similar to the patients reported by Gregersen et al. (13) and Naylor et al. (23), with nonketotic dicarboxylic aciduria. We believe that our patients have competent transport of long chain fatty acids into the mitochondria and subsequent ability to $\beta$-oxidize them to the medium chain length. Impairment of further $\beta$-oxidation to shorter lengths may result in the accumulation of medium chain length acids in the mitochondria. They may escape from the mitochondria and be metabolized by the $\omega$ and $\psi$-pathways available in the cytoplasm and give rise to the dicarboxylic acids and the $\psi$-derivatives respectively. Unusually high octanoic acid concentrations may inhibit lipolysis of depot fat and reduce the input of long chain substrate during an attack. In addition, unavailability of the glycerol moiety as a gluconeogenic substrate probably contributes to the hypoglycemia. In intensely catabolic circumstances where normal children metabolize fat chiefly by both the $\beta$ and $\omega$-pathways, our patients may rely on the $\omega$ - and $\psi$-pathways due to an incompletely useful $\beta$-pathway. Increased $\psi$-oxidation capability may furthermore be induced by the chronically high medium chain substrate levels in our patients. The defect becomes of clinical significance only when prolonged fasting is coupled with increased energy requirements (from fever due to nonspecific infection), a situation in which fatty acid $\beta$-oxidation is normally greatly increased.

The mechanism of the hypoglycemia is not clear but may be secondary to sequestration of coenzyme A, a requirement for activation of pyruvic carboxylase, the first step in gluconeogenesis. As well, there is diminished ketone body production to provide an alternate fuel source. The children with starvation ketonuria and normoglycemia, in contrast to patients 1 and 2, had large amounts of 3-hydroxybutyric and acetoacetic acids. Young infants are normally at increased risk for all types of fasting hypoglycemia due to the disparity between the mass of the glucose requiring organ (the nervous system) and that of those organs involved either in new glucose formation or in provision of alternate energy sources (liver, muscle, and adipose tissue). We predict that these childrens' liability to hypoglycemia will decrease with age although symptoms due to compromised fatty acid oxidation in muscle may increase with growth of that organ. The prominent role of fat as an energy substrate in infants is emphasized (24).

The present cases are reported to encourage collection of urine and serum for gas chromatographic-mass spectrometric analysis from children with unexplained hypoglycemia, especially those with low insulin levels and minimal ketonuria. $\psi$-Hydroxyacid excretion, in low but easily measurable concentrations, persisted between attacks in these children; thus, the examination of urine specimens after recovery or after challenge with triglyceride loads may be of diagnostic value in other patients suspected of having a similar disorder.

\section{REFERENCES AND NOTES}

1. Bjorkhem. I. and Danielsson, H.: $\omega$ and $(\omega-1)$-Oxidation of fatty acids by rat liver microsomes. Eur. J. Biochem.. 17: 450 (1970).
2. Bjorkhem, I.: On the quantitative importance of omega-oxidation of fatty acids J. Lipid Res., 19: 585, (1978).

3. Bjorkhem, I, and Hamberg, M.: $\omega$-Oxidation of fatty acids. II. Enzymatic oxidoreduction of 17-hydroxystearic acid. J. Biol. Chem., 246: 7417 (1971).

4. Chalmers, R. A.. Lawson, A. M., Whitelaw, A., and Purkiss, P.: Organic acids in Reye's like syndrome: similarities with Jamaican Vomiting Sickness. Lancet, 1: 1155 (1977)

5. Chalmers, R. A. and Lawson, A. M.: Dicarboxylic aciduria as an indicator of defective $\beta$-oxidation in twin siblings with a vomiting sickness and hypoglycaemia similar to Jamaican vomiting sickness. Biochem. Soc. Trans., 6: 111 (1978).

6. Chalmers, R. A., Lawson, A. M., Whitelaw, A., and Purkiss, P.: Twin siblings with a Reye's like syndrome associated with abnormal organic aciduria, hypoglycemia, diarrhea, and vomiting with close similarities to Jamaican vomiting sickness. Pediatr. Res., 14: 1097 (1980).

7. Crocker, J. F. S. and Bagnell, P. G.: Reye's syndrome: a clinical review. Can. Med. Assoc. J., 124: 375 (1981).

8. DeVivo, D. C.: Reye's Syndrome: a metabolic response to an acute mitochondrial insult? Neurology, 106: 105 (1978)

9. Dosman, J., Crawhall, J. C., Klassen, G. A., Mamer, O. A., and Neumann, P.: Urinary excretion of $\mathrm{C} 6-10$ dicarboxylic acids in glycogen storage disease type I and III. Clin. Chim. Acta, 61: 93 (1974).

10. Geber, N., Dickinson, R. G., Harland, B. R. C., Lynn, R. K., Houghton, D., Antonias, J. I., and Schimschock, J. C.: Reye-like syndrome associated with valproic acid therapy. J. Pediatr., 95: 141 (1979).

11. Gregersen, N., Lauritzen, R., and Rasmussen, K.: Suberylglycine excretion in the urine from a patient with dicarboxylic aciduria. Clin. Chim. Acta, 70: 417 (1976).

12. Gregersen, N.: Specific and sensitive method for the determination of $C_{6}-C_{10}$ dicarboxylic acids in serum and urine by selected ion monitoring. J. Chromatogr., 162: 377 (1979).

13. Gregersen, N., Rosleff, F., Kolvraa, S., Hobolth, N., Rasmussen, K., and Lauritzen, R.: Non-ketotic $\mathrm{C}_{6}-\mathrm{C}_{10}$ dicarboxylic aciduria: biochemical investigation of two cases. Clin. Chim. Acta, 102: 179 (1980).

14. Hillman, D. A. and Colle, E.: Plasma growth hormone and plasma insulin responses in children with growth retardation. Amer. J. Dis. Child., 117: 636 (1969)

15. Kam, W., Kumaran, K., and Landau, B. R.: Contribution of omega-oxidation to fatty acid oxidation by liver of rat and monkey. J. Lipid Res. 19: 591 (1978)

16. Karpati, G., Carpenter, S., Engel, A. G., Watters, G., Allen, J., Rothman, S. Klassen, G., and Mamer, O.: The syndrome of systemic carnitine deficiency. Neurology, 25: 16 (1975)

17. Lawson, A. M. and Chalmers, R. A.: Identification of 5-hydroxyhexanoic acid in the urine of twin siblings with a syndrome having close similarities to Jamaican Vomiting Sickness. Biochem. Society Trans., 6: 108 (1978).

18. Laurell, S. and Tibbling, G.: Colorimetric micro determination of free fatty acids in plasma. Clin. Chim. Acta, 16: 57 (1967).

19. Mamer, O. A., Crawhall, J. C., and Tjoa, S. S.: The identification of urinary acids by coupled gas chromatography-mass spectrometry. Clin. Chim. Acta 32: $171(1971)$.

20. Mamer, O. A., Montgomery, J. A., and Colle, E.: Profiles in altered metabolism. III: $(\Omega-1)$-Hydroxyacid excretion in a case of episodic hypoglycemia. Biomed. Mass Spectrom., 7: 53 (1980).

21. Mortensen, P. B. and Gregersen, N.: Medium chain triglyceride medication as a pitfall in the diagnosis of non-ketotic $\mathrm{C} 6-\mathrm{C} 10$ dicarboxylic aciduria. Clin Chim. Acta, 102: 33 (1980)

22. Mortensen, P. B.: Inhibition of fatty acid oxidation by valporate. Lancet, 2: 856 (1980).

23. Naylor, E. W., Mosovich, L. L., Guthrie, R., Evans, J. E., and Tieckelmann, H. Intermittent Non-ketotic Dicarboxylic Aciduria in Two Siblings with Hypoglycaemia: An Apparent Defect in $\beta$-Oxidation of Fatty Acids. J. Inher. Metab. Dis., 3: 19 (1980).

24. Senior, B. and Wolfsdorf, J. I.: Hypoglycemia in children. Ped. Clin. N. Amer. 26: 171 (1979).

25. Shigematsu, Y., Momoi, T., Masakatsu, S., and Suzuki, Y.: ( $\omega$-1)-Hydroxymonocarboxylic acids in urine of infants fed medium-chain triglycerides. Clin. Chem., 27: 1661 (1981).

26. Tanaka, K., Kean, E. A., and Johnson, B.: Jamaican Vomiting Sickness: biochemical investigation of two cases. N. Engl. J. Med., 295: 462 (1976).

27. Tanaka, K.: "Jamaican Vomiting Sickness" In: P. J. Vinken and G. W. Bruyn Eds.: Intoxications of the Nervous System, Part II. pp 529-535 (North Holland Publishing Co., New York, 1979).

28. Van Caille, M., Morin, C. L., Roy, C. C., Geoffroy, G., and McLaughlin, B Reye's syndrome relapses and neurologic sequelae. Pediatrics, 59: 245 (1977).

29. Informed consent to administer the triglyceride loads was obtained from the parents.

30. The technical assistance of Mr. Luc Choinière is gratefully acknowledged.

31. Requests for reprints should be addressed to: Dr. Eleanor Colle, Division of Endocrinology, Montreal Children's Hospital, 2300 Tupper, Montreal, Quebec. Canada.

32. This research was supported by the Medical Research Council of Canada.

33. Received for publication April 13, 1981 .

34. Accepted for publication June 1, 1982. 